\title{
Utilização da manutenção centrada em confiabilidade (MCC) em embaladora à
} vácuo de alimentos

\section{Reliability-maintenance maintenance (RMC) using in food vacuum packaging}

Article Info:

Article history: Received 2021-02-22 / Accepted 2021-04-06 / Available online 2021-04-06

doi: $10.18540 /$ jcecvl7iss2pp12021-01-14e

Lucas Benini

ORCID: https://orcid.org/0000-0002-5850-1399

Departamento de Engenharia Mecânica, TEM - Universidade Federal Fluminense, UFF, Brasil

E-mail: lucasbenini@id.uff.br

Alexandre Santos

ORCID: https://orcid.org/0000-0003-0146-896X

Departamento de Engenharia de Produção e Mecânica, DEP - Universidade Federal de Viçosa,

UFV, Brasil

E-mail: alexanddr@hotmail.com

\section{Resumo}

A manutenção centrada na confiabilidade (MCC) trata-se de uma abordagem que tem foco na prevenção de falhas, cujas consequências geram problemas de maior gravidade. Esse método tem sido empregado na gestão da manutenção em diversos setores industriais. No setor alimentício, as embaladoras a vácuo têm um papel de extrema importância no processo produtivo, pois garantem a selagem e conservação dos produtos. Na empresa avaliada, as embaladoras a vácuo apresentam acentuado histórico de falhas, revelando que a atual metodologia de manutenção não atende as condições esperadas para estes equipamentos. O objetivo deste trabalho é aplicar a metodologia de manutenção centrada na confiabilidade em embaladoras a vácuo de uma empresa do setor alimentício, visando melhorar os índices de confiabilidade, de disponibilidade e os custos. Para tanto, os passos metodológicos seguiram o seguinte desdobramento: (i) seleção do sistema e coleta de informações, (ii) análise dos modos e efeitos de falha (iii) seleção de funções significantes (iv) avaliação de atividades aplicáveis ( $v$ ) avaliação de efetividade das atividades. A aplicação da manutenção centrada na confiabilidade permitiu aplicar melhorias em exploração da função da máquina, uso do histórico do setor, conhecimento das falhas e aumentou o índice de confiabilidade e disponibilidade. Os custos de manutenção foram diminuídos em torno de 55\%.

Palavras-chave: Manutenção. Confiabilidade. Redução de falhas. Redução de custos. Indústria de alimentos.

\begin{abstract}
Reliability-centered maintenance (RCM) is an approach that focuses on failure prevention, its consequences generate more serious problems. This RCM method has been used in the management of maintenance in several industrial sectors. In the food sector, vacuum packaging machines play an extremely important role in the production process, as they guarantee the sealing and conservation of products. In this study, vacuum packers have a long history of failures in the company, revealing that the current maintenance methodology does not meet the conditions expected for this equipment. The objective of this work is to apply the RCM methodology in a vacuum packaging machines of a company in the food sector, aiming to improve the reliability, availability and costs. The methodological steps followed the following breakdown: (i) system selection and information collection, (ii) analysis of failure modes and effects (iii) selection of significant functions (iv) evaluation of applicable activities (v) evaluation effectiveness of activities. The application of maintenance centered on reliability allowed improvements to be made in the
\end{abstract}


machine's function exploration, in the use of the sector's history, in the knowledge of failures and to increase the reliability and availability index. Maintenance costs were reduced by around 55\%.

Keywords: Maintenance. Reliability. Failure reduction. Cost reduction. Food industry.

\section{Introdução}

A competitividade e as constantes mudanças na economia impõem às empresas reduzir seus custos e atuar de forma responsável em relação à segurança e ao meio ambiente, o que é um desafio para o setor de manutenção (Bandeira et al., 2016). Por este motivo, os gestores da manutenção devem considerar a visão estratégica da empresa ao selecionar metodologias de manutenção que atendam a esse desafio (Silva, 2004). As práticas desenvolvidas na gestão da manutenção têm sido motivadas pela busca da excelência e redução dos custos operacionais, onde o aumento da confiabilidade de equipamentos é obrigatório nos meios de manufatura (Caiado et al., 2015).

Dentre as metodologias empregadas na gestão da manutenção, as mais utilizadas são a manutenção produtiva total TPM (Total Productive Maintenance) e a manutenção centrada na confiabilidade MCC (ou Reability Centered Maintanance, RCM) (Baran, 2011). A MCC é baseada nas normas SAE JA10 11 (1999) e IEC 60300-3-11 (1999) e considera dados estatísticos e o histórico de manutenção para atingir resultados satisfatórios. O principal objetivo da MCC é criar rotinas estratégicas de manutenção que assegurem que os equipamentos continuarão desempenhando suas funções pré-determinadas da forma mais econômica possível na planta industrial (Wilmeth e Ursey, 2000; Fogliatto e Ribeiro, 2009; Mendes, 2011).

A MCC tem sido abordada em diversos trabalhos: Flemming (1999) discorreu sobre a MCC em 6 casos nas indústrias de siderurgia, offshore e petroquímica; Lucatelli (2002) empregou a MCC em equipamentos médicos hospitalares; Sellitto et al. (2002) aplicaram a MCC no desenvolvimento de uma metodologia para gerenciamento de risco, a qual foi aplicada em um ônibus urbano; Alkaim (2003) propôs a utilização da MMC em equipamentos sistemas elétricos; Silva (2004) utilizou a MMC no setor de pintura de uma montadora automobilística; Baran (2011) utilizou o MCC em um desbobinador de alumínio de um processo industrial de laminação; Mendes (2011) aplicou a MCC em uma multinacional no setor de tintas e corantes; Caiado et al. (2015) empregaram a MCC em uma ferramenta de perfuração utilizada no setor de óleo e gás; Cerveira e Sellitto (2015) aplicaram a MCC em um forno elétrico a indução em uma fundição de aços especiais; Pereira (2015) fez uso da MCC em uma embaladora automática de refrescos (30 g); Bandeira et al. (2016) empregaram o MCC em um sistema de refrigeração à base de água de um shopping center; Rosa (2016) utilizou a MCC em equipamentos transportadores utilizados na montagem em uma linha de produção automotiva; Amaral (2017) utilizou a MCC para a manutenção de navios; Silva (2018) aplicou o MCC em um equipamento de moer de grãos; Araldi (2019) empregou a MCC em um centro de usinagem comandado numericamente.

Apesar de vários trabalhos reportados na literatura, poucos são aqueles onde a MCC é utilizada no setor de alimentos. Belinelli (2015) destaca que a ocorrência de falhas em equipamentos e máquinas de um processo produtivo de alimentos pode acarretar prejuízos para a indústria de alimentos, pois estas falhas podem causar contaminação por resíduos químicos, físicos ou biológicos. Por este motivo, indústrias do setor alimentício devem buscar excelência na gestão da manutenção do seu maquinário, pois as consequências de falhas podem ser desde atrasos na entrega de produtos até o impacto negativo da imagem pública da empresa, devido a contaminação acidental do produto. Dentre os vários equipamentos utilizados em linhas de produção de alimentos destacase a embaladora do tipo à vácuo, que deve possuir uma elevada confiabilidade e garantir segurança sanitária aos produtos.

A empresa analisada neste trabalho possui um mix de 72 produtos alimentícios, sendo que o histórico de falhas revela que há deficiências no processo de embalagem de um de seus principais produtos. Estas deficiências são originadas principalmente por uma manutenção deficiente e que prejudica os resultados da empresa. A embaladora à vácuo é utilizada na embalagem de um produto 
que foi responsável por cerca de $25 \%$ do faturamento da empresa em 2019. Essa embaladora apresentou 27 falhas ao longo de 2019, ocasionando um tempo de parada de máquina em torno de 50 horas mensais em média. Por se tornar um gargalo na linha de produção, a ações para reduzir esses indicadores são obrigatórias diante da constante busca por redução de custos e otimização de processos na indústria alimentícia. As embaladoras à vácuo, além de garantirem a selagem e conservação do produto exigida pela legislação, são responsáveis pela embalagem final e apresentação do produto, atuando como parte intrínseca do marketing do produto final, ao considerar a sua apresentação para os clientes em pontos de vendas e gôndolas de mercados.

O objetivo deste trabalho é aplicar os princípios de manutenção centrada na confiabilidade (MCC) em uma embaladora a vácuo na linha de produção de uma empresa do setor alimentício, visando reduzir os custos com trocas de componentes, elevar a confiabilidade e disponibilidade da máquina e reduzir as perdas no processo de embalagem para a empresa.

\section{Manutenção centrada na confiabilidade (MCC)}

A MCC é uma metodologia de manutenção que reúne diferentes técnicas de engenharia para estudar um equipamento em detalhes. Por meio dela, é analisado como a máquina pode falhar e, é definido a estratégia de manutenção mais eficaz, incluído ações corretivas, preventivas, preditiva, que quando executadas, irão reduzir a probabilidade de falhas em um equipamento, bem como seus custos de manutenção, tendo como principal objetivo que as máquinas realizem suas funções especificadas (Kardec e Nascif, 2009).

O diferencial da MCC em relação as demais metodologias de manutenção é a sua normatização, através da norma SAE JA 1011 (1999) e IEC 60300-3-11 (1999) que tratam todos requisitos para implantação desse método. O objetivo dessa metodologia é a redução do custo do ciclo de vida do ativo aplicando cada tipo de manutenção nos componentes do ativo de maneira estruturada. A Tab. 1 compara as características da MMC com a manutenção tradicional.

Tabela 1 - Comparação da manutenção tradicional com a MCC (Siqueira, 2014).

\begin{tabular}{l|l|l}
\hline Característica & Manutenção tradicional & MCC \\
\hline Foco & Equipamento & Função \\
\hline Objetivo & Manter o equipamento & Preservar função \\
\hline Atividades de manutenção & $\begin{array}{l}\text { Definidas com base no que pode } \\
\text { ser feito }\end{array}$ & $\begin{array}{l}\text { Definidas com base no que } \\
\text { deve ser feito }\end{array}$ \\
\hline Dados históricos & Pouca ênfase & Muita ênfase \\
\hline Metodologia & Empírica & Estruturada \\
\hline Combate & Deterioração do equipamento & Consequências das falhas \\
\hline
\end{tabular}

O processo de implantação da MCC na manutenção de um equipamento ou sistema pode ser resumido em sete etapas (Moubray, 1997; Siqueira, 2009; Smith e Hinchcliffe, 2004; Siqueira 2014):

- Etapa 1: Seleção do Sistema e Coleta de informação;

- Etapa 2: Análise dos Modos e Efeitos de Falha;

- Etapa 3: Seleção de Funções Significantes;

- Etapa 4: Avaliação de Atividade Aplicável;

- Etapa 5: Avaliação de Efetividade das Atividades;

- Etapa 6: Seleção de Atividade Aplicáveis e Efetivas;

- Etapa 7: Definição da Periodicidade das Atividades.

Destaca-se que que as Etapas 4, 5 e 6 podem ser condensadas em uma única etapa (que foi o caso deste trabalho). 


\section{Procedimentos Metodológicos}

Este trabalho foi efetuado em uma unidade de uma empresa do setor alimentício localizada em Minas Gerais. Nesta unidade, que possui um mix de 72 produtos, o produto determinado para aplicação da metodologia MCC na linha de produção representa em torno de $25 \%$ do faturamento e atende tanto o mercado interno quanto o externo. Para o estudo, os dados referentes aos planos de manutenção e custos foram coletados do sistema ERP (Enterprise Resource Planning) da empresa. Para a aplicação da MCC na embaladora à vácuo foi designada uma equipe interdisciplinar, composta por três colaboradores do setor de Planejamento e Controle de Manutenção (PCM), Supervisão e Engenharia de Manutenção da unidade. Deve-se destacar que atualmente a manutenção das máquinas é, em grande parte, baseada na manutenção preventiva.

No contexto desta unidade, a manutenção considera somente a experiência dos profissionais da área e fatores como o tempo de uso e o ambiente em que a máquina está alocada não são observados. A aplicação da MCC na embaladora a vácuo de alimentos seguiu as etapas descritas recomendadas na seção 2. A Fig. 1 representa esquematicamente os desdobramentos da metodologia aplicada neste trabalho.

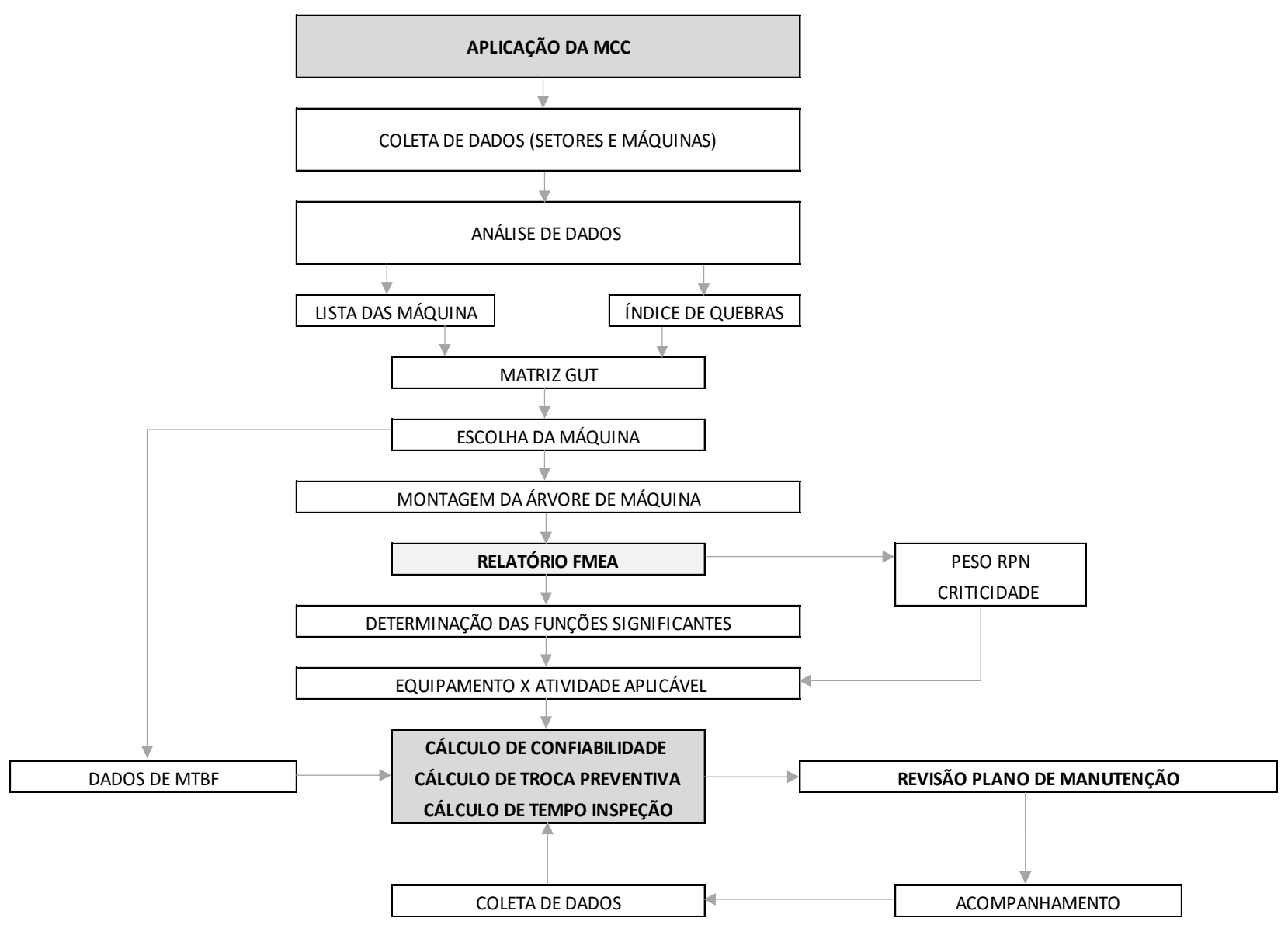

Figura 1 - Metodologia de aplicação da MCC na embaladora à vácuo.

\subsection{Seleção do sistema e coleta de informações}

A seleção do equipamento analisado levou em consideração o número de paradas de máquinas da linha de produção mais produtiva da empresa. Inicialmente nesta linha foi definido o grupo de equipamentos críticos e utilizada a ferramenta de gestão denominada matriz GUT. A matriz GUT, sigla para Gravidade, Urgência e Tendência, é uma ferramenta de gestão utilizada para priorização de tarefas. Ao identificar a urgência, a gravidade e a tendência de comportamento de cada problema na empresa, ajuda o administrador a decidir quanto ao que fazer primeiro (Correia Júnior, 2016). A 
Tab. 2 apresenta as ponderações da matriz GUT que demonstra a embaladora à vácuo como o equipamento mais crítico segundo a matriz.

Tabela 2 - Pontuação GUT dos equipamentos.

\begin{tabular}{c|c|c|c|c|c}
\hline Máquina & $\mathbf{N}^{\circ}$ de falhas & $\mathbf{G}$ & $\mathbf{U}$ & $\mathbf{T}$ & Pontuação GUT \\
\hline Embaladora à vácuo A & 27 & 4 & 4 & 2 & 32 \\
\hline Depiladeira B & 23 & 5 & 3 & 2 & 30 \\
\hline Depiladeira A & 15 & 5 & 3 & 2 & 30 \\
\hline Tanque de resfriamento & 14 & 5 & 4 & 1 & 20 \\
\hline Embaladora à vácuo B & 17 & 4 & 4 & 1 & 16 \\
\hline Misturadeira & 14 & 5 & 3 & 1 & 15 \\
\hline Embutideira A & 47 & 2 & 3 & 2 & 12 \\
\hline Emulsificador & 20 & 5 & 1 & 2 & 10 \\
\hline Embutideira C & 56 & 3 & 3 & 1 & 9 \\
\hline Ensacadora & 13 & 2 & 2 & 2 & 8 \\
\hline Transportador & 9 & 4 & 2 & 1 & 8 \\
\hline Embutideira B & 72 & 2 & 3 & 1 & 6 \\
\hline
\end{tabular}

Em seguida, foi efetuada a coleta de informações do sistema. A máquina em questão é uma embaladora a vácuo, responsável por vedar os pacotes por meio de termo fusão. Uma esteira leva os pacotes carregados para dentro da câmara de vácuo e selagem. Com os pacotes sob a câmara, os atuadores pneumáticos descem a tampa até fechá-la, e inicia-se a retirada de ar através de um conjunto de bomba à vácuo. Posteriormente, resistência elétricas são ligadas por poucos segundos, fundindo o plástico e selando o produto. Por fim, uma pequena válvula de alívio libera a entrada do ar atmosférico, abrindo a tampa e fazendo a esteira descarregas o pacote de produto selados e iniciando um novo ciclo. A Fig. 2 esquematiza o diagrama organizacional da máquina, que foi dividida em três conjuntos, subdivididos em equipamentos, bem como a embaladora a vácuo.
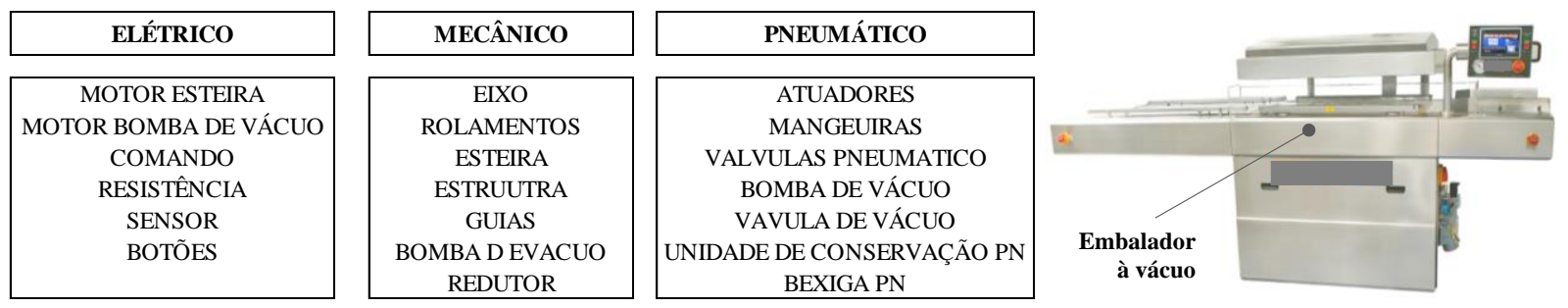

Figura 2 - Diagrama organizacional da máquina: conjuntos e equipamentos da embaladora à vácuo.

\subsection{Análise dos modos e efeitos de falha}

Nessa etapa foi aplicada a ferramenta de análise FMEA (Failure Mode and Effects Analysis). A Associação Brasileira de Norma Técnicas (ABNT), na norma NBR 5462 (1994), a traduz como sendo Análise dos Modos de Falha e seus Efeitos. O FMEA é um documento onde se segue uma sequência de passos lógicos: divisão da máquina em conjuntos e componentes, identificando suas funções; identificação das falhas, traçando o efeito dessa falha e suas causas; avaliação de risco caso a falha ocorra (Moubray, 1999). Com base na divisão da máquina (Fig. 3), foram definidas as funções de cada equipamento dos subconjuntos, e definidas suas possíveis falhas e os efeitos que estas podem gerar. Por fim, foram determinadas as possíveis causas dessas falhas e quais seus riscos. O preenchimento do FMEA foi efetuado por uma equipe de três colaboradores. As Tab. 3 apresenta o excerto do relatório do FMEA correspondente ao conjunto elétrico. 
Tabela 3 - Excerto do relatório do FMEA do conjunto elétrico da embaladora à vácuo.

\begin{tabular}{|c|c|c|c|c|c|c|c|c|c|c|c|c|c|c|c|c|c|c|}
\hline \multirow{2}{*}{\multicolumn{2}{|c|}{$\begin{array}{l}\text { SISTEMA: } \\
\text { EQUIPE: }\end{array}$}} & & \multirow{2}{*}{\multicolumn{3}{|c|}{\begin{tabular}{|c|} 
local \\
Colaborador 1, Colaborador 2 e Colaborador 3
\end{tabular}}} & \multirow{2}{*}{\multicolumn{2}{|c|}{$\begin{array}{l}\text { DATA EXECUÇÃO: } \\
\text { VERSÃO: }\end{array}$}} & \multirow{2}{*}{\multicolumn{3}{|c|}{ data }} & & \multirow{2}{*}{\multicolumn{7}{|c|}{$\begin{array}{c}\text { Função da Máquina: } \\
\text {-- }\end{array}$}} \\
\hline & & & & & & & & & & & & & & & & & & \\
\hline \multicolumn{4}{|c|}{ PONTO DE FALHA } & \multicolumn{3}{|c|}{ ANÁLISE DA FALHA } & \multicolumn{5}{|c|}{ AVALIAÇÃO DE RISCO } & \multicolumn{7}{|c|}{ MATRIZ DE CRITICIDADI } \\
\hline CONJUNTO & EQUIPAMENTO & $\begin{array}{l}\text { FUNÇ̃̃O DO } \\
\text { EQUIPAMENTO }\end{array}$ & COMPONENTES & MODO DE FALHA & EFEITO DE FALHA & CAUSA DA FALHA & 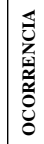 & 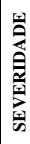 & 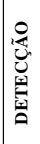 & Z & 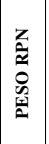 & 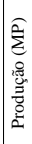 & 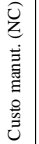 & 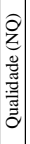 & 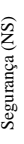 & 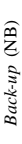 & 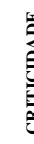 & \\
\hline \multirow{14}{*}{$\frac{0}{0}$} & \multirow{4}{*}{ MOTOR DA ESTEIRA } & \multirow{4}{*}{$\begin{array}{l}\text { Movimentar a esteira } \\
\text { principal }\end{array}$} & Rolamento & Ruido & travamento & desgaste & 4 & 8 & 6 & 192 & $43 \%$ & \multirow{4}{*}{1} & \multirow{4}{*}{3} & \multirow{4}{*}{2} & \multirow{4}{*}{2} & \multirow{4}{*}{2} & \multirow{4}{*}{24} & \multirow{4}{*}{ \#\# } \\
\hline & & & Tampa & trinca & quebra & desbalanciamento & 2 & 6 & 7 & 84 & $19 \%$ & & & & & & & \\
\hline & & & Eixo & vibração & quebra & desalinhamento & 4 & 7 & 5 & 140 & $31 \%$ & & & & & & & \\
\hline & & & Ventilador & aquecimento & curto-circuito & quebra das pás da hélice & 2 & 5 & 6 & 60 & $13 \%$ & & & & & & & \\
\hline & \multirow{4}{*}{$\begin{array}{l}\text { MOTOR DA BOMBA } \\
\text { DE VÁCUO }\end{array}$} & \multirow{4}{*}{$\begin{array}{l}\text { Movimentar conjunto } \\
\text { da bomba de vacuo }\end{array}$} & Eixo & vibração & quebra & sobre carga & 1 & 7 & 5 & 35 & $8 \%$ & & & \multirow{4}{*}{3} & & & \multirow{4}{*}{108} & \\
\hline & & & Rolamentos & ruido & travamento & desgaste & 2 & 6 & 5 & 60 & $13 \%$ & \multirow{3}{*}{2} & \multirow{3}{*}{3} & & \multirow{3}{*}{3} & \multirow{3}{*}{2} & & \multirow{3}{*}{8 \#\# } \\
\hline & & & $\begin{array}{l}\text { Engrenagem de } \\
\text { corrente }\end{array}$ & $\begin{array}{c}\text { deteriorização dos } \\
\text { dentes }\end{array}$ & perda de tração & falta de lubrificante & 4 & 8 & 4 & 128 & $29 \%$ & & & & & & & \\
\hline & & & corrente & folga & quebra & falta de tensionamento & 4 & 8 & 3 & 96 & $21 \%$ & & & & & & & \\
\hline & \multirow{4}{*}{ COMANDO } & \multirow{4}{*}{$\begin{array}{l}\text { Distribuir Energia e } \\
\text { controlar a máquina }\end{array}$} & Disjuntor & \multirow{4}{*}{ Aquecimento } & & & & & & & & & & & & & & \\
\hline & & & $\begin{array}{c}\text { Contator } \\
\text { Bornes }\end{array}$ & & Não comuta & sobre carga & 1 & 6 & 5 & 30 & $7 \%$ & 2 & 2 & 3 & 2 & 2 & 96 & \#\# \\
\hline & & & Cabos & & & & & & & & & & & & & & & \\
\hline & & & botoes & & & & & & & & & & & & & & & \\
\hline & RESISTÊNCIA & $\begin{array}{l}\text { Selar o pacote por } \\
\text { termofusão }\end{array}$ & Resistência & Não aquece & Não sela o pacote & Quebra & 8 & 7 & 8 & 448 & \#\#\# & 1 & 3 & 1 & 3 & 2 & 18 & \# \\
\hline & SENSOR & $\begin{array}{l}\text { Identificar posições na } \\
\text { máquina }\end{array}$ & Sensor & Não liga & Não comuta & Queima & 4 & 5 & 7 & 140 & $31 \%$ & 3 & 3 & 3 & 3 & 2 & 162 & \#\# \\
\hline
\end{tabular}

\subsection{Seleção das funções significantes}

$\mathrm{Na}$ terceira etapa, foram avaliadas quais das funções dos componentes descritas no FMEA serão abordadas pelo MCC. A Tab. 4 apresenta a classificação das funções significantes, classificadas de acordo com os critérios mostrados descritos por Siqueira (2014), que afirma que uma função considerada significante deverá ser tratada se ela já tem uma atividade de manutenção, ou se ela afeta a segurança, meio ambiente, operação ou economia do processo.

Tabela 4 - Classificação das funções significantes dos componentes da embaladora à vácuo.

\begin{tabular}{|c|c|c|c|c|c|c|c|}
\hline CONJUNTO & EQUIPAMENTO & FUNÇÃO & $\begin{array}{l}\text { Perda da função tem } \\
\text { efeito na segurança } \\
\text { ou meio ambiente? }\end{array}$ & $\begin{array}{c}\text { Perda da função } \\
\text { tem efeito na } \\
\text { operação? }\end{array}$ & $\begin{array}{c}\text { Perda da função tem } \\
\text { efeito na economia } \\
\text { do processo? }\end{array}$ & $\begin{array}{c}\text { A função já tem } \\
\text { terefa de } \\
\text { manutenção? }\end{array}$ & $\underline{R E S U L T A D O}$ \\
\hline \multirow{5}{*}{ Eétrico } & MOTOR DA ESTEIRA & Movimentar a esteira principal & $\mathrm{N}$ & $\mathrm{S}$ & $\mathrm{N}$ & $\mathrm{S}$ & SIGNIFICANTE \\
\hline & $\begin{array}{l}\text { MOTOR DA BOMBA } \\
\text { DE VÁCUO }\end{array}$ & Movimentar conjunto da bomba de vácuo & $\mathrm{N}$ & $\mathrm{S}$ & $\mathrm{N}$ & $\mathrm{N}$ & SIGNIFICANTE \\
\hline & COMANDO & Distribuir energia e controlar a máquina & $\mathrm{N}$ & $\mathrm{S}$ & $\mathrm{N}$ & $\mathrm{N}$ & SIGNIFICANTE \\
\hline & RESISTÊNCIA & Selar o pacote por termofusão & $\mathrm{N}$ & $\mathrm{S}$ & $\mathrm{N}$ & $\mathrm{S}$ & SIGNIFICANTE \\
\hline & SENSOR & Identificar posições na máquina & $\mathrm{N}$ & $\mathrm{N}$ & $\mathrm{N}$ & $\mathrm{N}$ & NÃO SIGNIFICANTE \\
\hline \multirow{6}{*}{ Mecânico } & EIXO & Transmissão de rotação & $\mathrm{N}$ & $\mathrm{N}$ & $\mathrm{N}$ & $\mathrm{N}$ & NÃO SIGNIFICANTE \\
\hline & ROLAMENTOS & Centrar o eixo e permitir rotação & $\mathrm{N}$ & $\mathrm{N}$ & $\mathrm{N}$ & $\mathrm{S}$ & SIGNIFICANTE \\
\hline & ESTEIRA & Transportar o produto ao longo da máquina & $\mathrm{N}$ & $\mathrm{S}$ & $\mathrm{N}$ & $\mathrm{S}$ & SIGNIFICANTE \\
\hline & GUIAS & Manter alinhamento entre partes & $\mathrm{N}$ & $\mathrm{N}$ & $\mathrm{N}$ & $\mathrm{N}$ & NÃO SIGNIFICANTE \\
\hline & BOMBA DE VÁCUO & Gerar vácuo na camara de selagem & $\mathrm{N}$ & $\mathrm{S}$ & $\mathrm{N}$ & $\mathrm{S}$ & SIGNIFICANTE \\
\hline & REDUTOR & Movimentar a esteira principal & $\mathrm{N}$ & $\mathrm{S}$ & $\mathrm{N}$ & $\mathrm{S}$ & SIGNIFICANTE \\
\hline \multirow{5}{*}{ Pneumático } & ATUADOR & Transmissão de rotação & $\mathrm{N}$ & $\mathrm{N}$ & $\mathrm{N}$ & $\mathrm{S}$ & SIGNIFICANTE \\
\hline & MANGUEIRAS & Centrar o eixo e permitir rotação & $\mathrm{N}$ & $\mathrm{N}$ & $\mathrm{N}$ & $\mathrm{N}$ & NÃO SIGNIFICANTE \\
\hline & VÁLVULA DE VÁCUO & Gerar vacuo na câmara de selagem & $\mathrm{N}$ & $\mathrm{S}$ & $\mathrm{N}$ & $\mathrm{S}$ & SIGNIFICANTE \\
\hline & UNIDADE DE & Movimentar a esteira principal & $\mathrm{N}$ & $\mathrm{N}$ & $\mathrm{N}$ & $\mathrm{N}$ & NÃO SIGNIFICANTE \\
\hline & BEXIGA DE SOLDA & Movimentar a esteira principal & $\mathrm{N}$ & $\mathrm{S}$ & $\mathrm{N}$ & $\mathrm{S}$ & SIGNIFICANTE \\
\hline
\end{tabular}

\subsection{Avaliação de atividade aplicável}

Nesta etapa são definidos os tipos de manutenção aplicável para cada função significante do equipamento. A definição do tipo de manutenção foi baseada nos critérios apresentados na Tab. 5 .

Tabela 5 - Seleção da Atividade Aplicável (adaptado de Teles, 2017).

\begin{tabular}{c|c|c|c|c}
\hline $\begin{array}{c}\text { Criticidade } \\
\text { do ativo }\end{array}$ & RPN $<\mathbf{6 5 \%}$ & $\begin{array}{c}\text { RPN de 30 a } \\
\mathbf{6 4 , 9 \%}\end{array}$ & $\begin{array}{c}\text { RPN de 15 a } \\
\mathbf{2 9 , 9 \%}\end{array}$ & $\begin{array}{c}\text { RPN }< \\
\mathbf{1 4 , 9 \%}\end{array}$ \\
\hline $\mathrm{A}$ & $\begin{array}{c}\text { Monitoramento } \text { on-line, } \\
\text { Preditiva, Inspeção }\end{array}$ & Preditiva, Inspeção & Preventiva & Corretiva \\
\hline $\mathrm{B}$ & Preditiva, Inspeção & Inspeção & Preventiva & Corretiva \\
\hline $\mathrm{C}$ & Preventiva & Corretiva & Corretiva & Corretiva \\
\hline
\end{tabular}


Para tanto, o RPN, a criticidade e a relevância de cada equipamento foram ponderados, resultando o tipo de manutenção indicada. Para a elaboração da matriz de criticidade dos equipamentos da máquina empregou-se a Eq. 1 (Teles, 2017):

Criticidade $=N P \times N C \times N Q \times N S \times N B$

onde NP é o critério de produção, NC é o critério de custo de manutenção, NQ é o critério de qualidade, NS é o critério de segurança e meio ambiente e NB é o critério de Back-Up; sendo que todos variam entre 0 e 3. De acordo com o resultado da Eq. 1, atribui-se um nível de criticidade, conforme Teles (2017), que classifica o nível de criticidade de acordo com a nota: de 0 a 55 como "Alta criticidade (A)", de 56 a 161 como "Média criticidade (B)" e de 162 a 243 de "Baixa criticidade (C)". Posteriormente cruzam-se os resultados RPN com o nível de criticidade dos equipamentos para selecionar o tipo de manutenção mais adequado para manter o ativo na sua função específica, conforme a Tab. 6.

Tabela 6 - Tipo de manutenção aplicável para cada componentes da embaladora à vácuo.

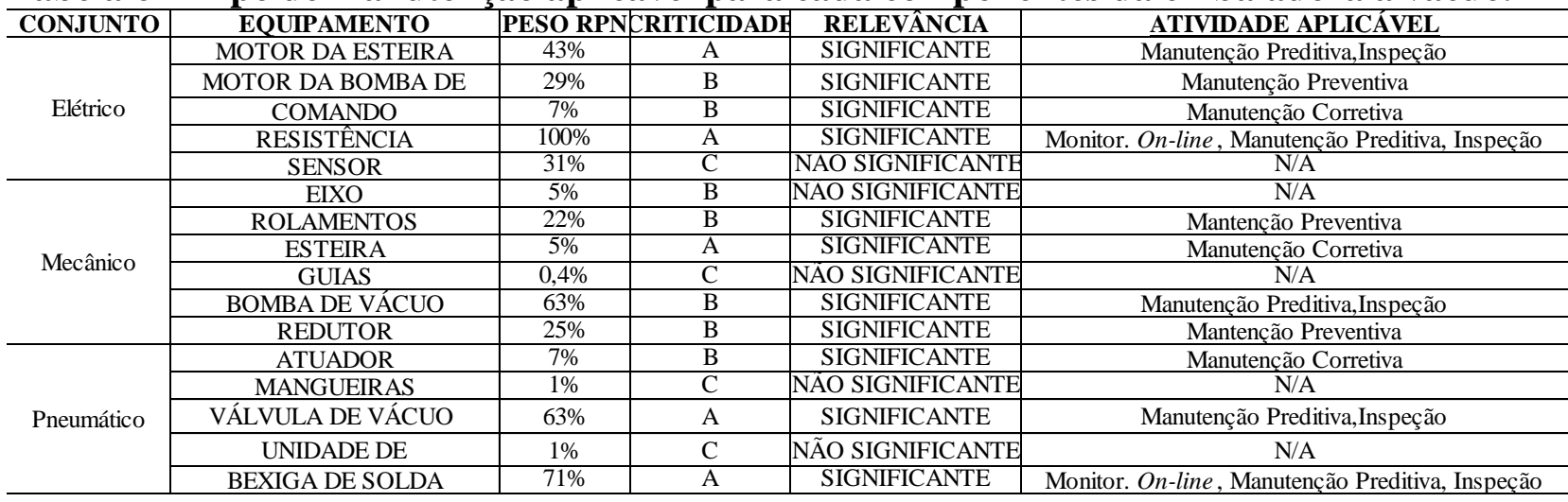

\subsection{Definição da periodicidade das atividades}

Nesta última etapa foi definida a periodicidades de manutenção das atividades utilizando-se dos os valores do tempo médio entre falhas (MTBF) do componente da máquina estudada (Eq. 2), que corresponde ao tempo de funcionamento de um componente até que a falha ocorra (Drumond, 2004).

$M T B F=\frac{\Sigma \text { Tempo em bom funcionamento }}{\text { Número de falhas }}$

onde MTBF é o tempo médio entre falhas [h]. A análise no número de paradas da máquina foi baseada nos dados coletados do histórico de paradas de máquina do período de fevereiro até novembro de 2019. Os dados de parada foram classificados em duas categorias: motivo da parada e tempo de parada (tempo que ficou inoperante na linha de produção). A Tab. 7 apresenta os MTBF em relação ao motivo da parada de máquina.

Tabela 7 - Classificação das funções significantes dos componentes da embaladora à vácuo.

\begin{tabular}{l|c|c}
\hline Tempo de bom funcionamento $[\mathbf{h}]$ & & $7.975,0$ \\
\hline MOTIVO & $\mathbf{N}^{\circ}$ de paradas & MTBF [h] \\
\hline Vácuo & 8 & 996,9 \\
\hline Resistência & 10 & 797,5 \\
\hline Redutor & 6 & 1329,2 \\
\hline Válvulas & 3 & $2.658,3$ \\
\hline
\end{tabular}


Com base nos resultados do MTBF (Tab. 7) foi calculada a confiabilidade para cada equipamento ligado a um motivo específico R(t) (Eq. 3). A taxa de falhas $\lambda$ foi dada pela Eq. (4) e o tempo futuro $t$ é empregado para estimar os tempos de trocas preventivas dos componentes, dado pela Eq. (5).

$R(t)=\mathrm{e}^{-\lambda \cdot t}$

$\lambda=\frac{1}{M T B F}$

$t=\ln (R) /-\lambda$

onde $R(t)$ é a confiabilidade [h], $\lambda$ é a taxa de falhas [\%] e $t$ corresponde ao tempo de trocas preventivas $[\mathrm{h}]$. Esses resultados, juntamente com a confiabilidade desejada de $85 \%$ (estipulada como objetivo deste trabalho) foram considerados para o cálculo do tempo futuro (Eq. 5), que foi usado para estimar os tempos de troca preventivas dos componentes, que resultou em $162 \mathrm{~h}$ (sendo que a taxa entre falhas foi de $\lambda=0,00100$ ). Com os resultados de MTBF, confiabilidade e tempo futuro relacionado a cada motivo de falha da máquina, usou-se a frequência de inspeção (70\% do valor de MTBF) e as substituições de peças em preventivas (Eq. 6), para estabelecer os tempos de inspeção e as trocas de componentes que foram utilizadas na atualização do plano de manutenção da máquina (Tab. 8).

$F t=0,85 x$ confiabilidade do componente

onde Ft é o tempo de substituições de peças em manutenções preventivas [h].

Tabela 8 - Tempos das atividades de manutenção.

\begin{tabular}{|c|c|c|c|c|c|}
\hline \multicolumn{2}{|c|}{ Tempo total no ano [h] } & 8.640 & \multirow{3}{*}{$\begin{array}{c}\text { Confiabilidade } \\
{[\mathrm{h}]} \\
\mathrm{R}(\mathrm{T})=85 \%\end{array}$} & \multirow{3}{*}{$\begin{array}{c}\text { Preventiva } \\
{[\mathrm{h}]} \\
0,85^{*} \text { conf. }\end{array}$} & \multirow{3}{*}{$\begin{array}{c}\text { Inspeção } \\
{[\mathrm{h}]} \\
\operatorname{MTBF}^{*} 0,7\end{array}$} \\
\hline Tempo de F & ncionamento [h] & 7.975 & & & \\
\hline MOTIVO & $\mathbf{N}^{\circ}$ PARADAS & MTBF [h] & & & \\
\hline Vácuo & 8 & 996,9 & 162,0 & 137,7 & 697,8 \\
\hline Resistência & 10 & 797,5 & 129,6 & 110,2 & 558,3 \\
\hline Redutor & 6 & $1.329,2$ & 216,0 & 183,6 & 930,4 \\
\hline Válvulas & 3 & $2.658,3$ & 432,0 & 367,2 & $1.860,8$ \\
\hline
\end{tabular}

Com atividades de manutenção anteriormente levantadas e categorizadas como relevantes, definida a melhor estratégia com as atividades aplicáveis, sumarizou-se os tempos estipulados de cada atividade na Tab. 9, denominada periodicidade das atividades. Cada equipamento possui uma determinada atividade aplicável, tempo de inspeção, recomendação de ação preditiva e horas de trocas de componentes em preventivas devidamente dimensionados.

Tabela 9 - Periodicidade das atividades.

\begin{tabular}{|c|c|c|c|c|c|}
\hline CONJUNTO & EQUIPAMENTO & ATIVIDADE APLICÁVEL & Inspeção [h] & Preditiva (Ação) & Preventiva [h] \\
\hline \multirow{3}{*}{ Elétrico } & MOTOR DA ESTEIRA & Manut. Preditiva, Inspeção & 930,4 & Vibração; Amperagem & 183,6 \\
\hline & \begin{tabular}{|c|} 
MOTOR DA BOMBA DE \\
VÁCUO \\
\end{tabular} & Manutenção Preventiva & & & 137,7 \\
\hline & RESISTÊNCIA & $\begin{array}{c}\text { Monitor. On-line, Manut. preditiva, } \\
\text { Inspeção }\end{array}$ & 558,3 & Termografia; Amperagem & 110,2 \\
\hline \multirow{3}{*}{ Mecânico } & ROLAMENTOS & Mantenção Preventiva & --------------- & 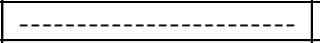 & 183,6 \\
\hline & BOMBA DE VÁCUO & Manut. Preditiva, Inspeção & 697,8 & Vibração; Termografia & $\mid---\cdot-\cdot--\cdot$ \\
\hline & REDUTOR & Mantenção Preventiva & |----------- & ------------------- & 183,6 \\
\hline \multirow[b]{2}{*}{ Pneumático } & VALVULA DE VÁCUO & Manut. Preditiva, Inspeção & 1860,8 & |------------------- & 367,2 \\
\hline & BEXIGA DE SOLDA & $\begin{array}{c}\text { Monitor. On-line, Manut. preditiva, } \\
\text { Inspeção }\end{array}$ & 558,3 & & \\
\hline
\end{tabular}




\section{Resultados e discussão}

Inicialmente são mostrados os resultados no período anterior a aplicação da MCC, de fevereiro a novembro de 2019, denominado PRÉ-MCC e posteriormente são abordados os resultados após a aplicação da MCC, de dezembro de 2019 em diante, denominados PÓS-MCC.

\subsection{Resultados período PRÉ-MCC}

A Fig. 3 mostra os resultados da confiabilidade e da disponibilidade da máquina ao longo do período PRÉ-MCC. Observa-se que a confiabilidade da máquina ao longo do período avaliado está abaixo de $60 \%$, com os índices mais baixos entre os meses de maio e agosto de 2019. Estes resultados de confiabilidade são evidenciados pelos índices de tempo médio entre falhas (MTBF), que demonstraram o número de paradas da máquina. Se observa na Fig. 3, em relação a disponibilidade, que somente nos meses de janeiro, de março e de setembro de 2019 o índice de disponibilidade esteve acima de $90 \%$. Considerando os resultados da Fig. 3, com índices de confiabilidade e de disponibilidade (na maior parte dos meses) abaixo de $60 \%$ no período PRÉMCC, pode-se constatar que o plano de manutenção utilizado não é compatível com os índices esperados para máquinas do setor alimentício.
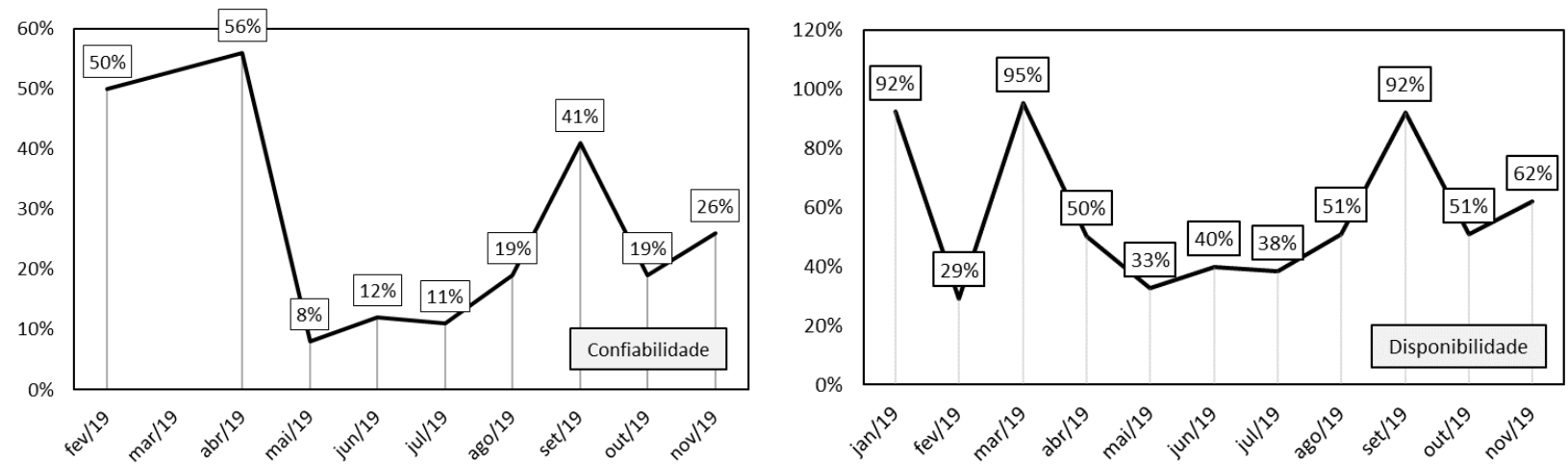

Figura 3 - Confiabilidade e disponibilidade da embaladora à vácuo - período PRÉ-MCC.

O plano de manutenção PRÉ-MCC da empresa tratava-se somente de manutenções preventivas. Na Fig. 4 está um excerto da lista de tarefas do plano de manutenção preventiva - onde se observa, no destaque desta figura, as peças que deverão ser trocadas - e um excerto do calendário de trocas das peças do plano de manutenção PRÉ-MCC, que expressa o tempo de troca em semanas.

\begin{tabular}{|c|c|c|c|c|c|}
\hline \multirow{2}{*}{\multicolumn{2}{|c|}{$\begin{array}{l}\text { Equipam. } \\
\text { GrpLisTar. }\end{array}$}} & & \multicolumn{3}{|c|}{ SELADORA } \\
\hline & & & & \multicolumn{2}{|c|}{ - PR - MECANICA } \\
\hline \multicolumn{6}{|c|}{ Síntese geral de operações } \\
\hline Oper & Sb... & - CenTrab & Cen. & Ctrl & Descrição \\
\hline 0010 & & MECAN & FABR & PM01 & TR. ROL. M \\
\hline 0020 & & MECAN & FABR & PM01 & TR. ROL. M \\
\hline 0050 & & MECAN & FABR & PM01 & \\
\hline 0060 & & MECAN & FABR & PM01 & TR. ROL./R \\
\hline 0070 & & MECAN & FABR & PM01 & TR. ROL. M \\
\hline
\end{tabular}

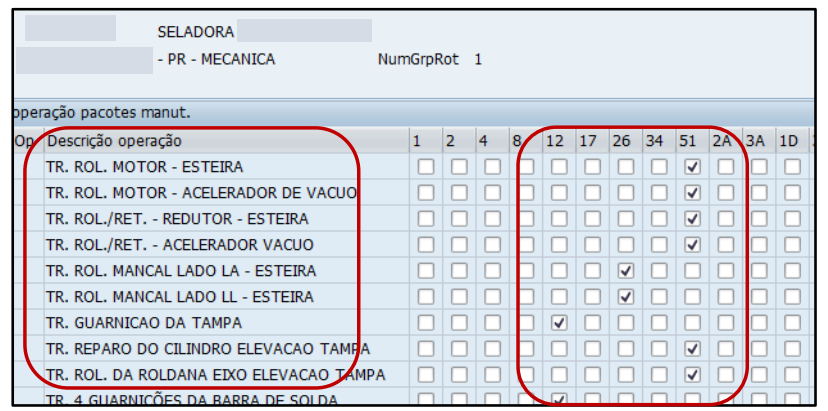

Figura 4 - Excerto da lista de tarefas (direita) e excerto do calendário de trocas (esquerda): plano de manutenção PRÉ-MCC.

Pode-se contatar que na lista de tarefas (Fig. 4) o plano de manutenção não está tratando as falhas da máquina. Atrelado a lista de tarefas, têm-se o calendário de trocas em relação a semana do ano. Observa-se nesse plano que a execução das operações de manutenção está concentrada nas semanas 12, 26 e 51 (destaque do calendário de trocas, Fig. 4). Conforme os resultados de disponibilidade da máquina (Fig. 3) a execução de todas as operações de manutenção não deve 
ocorrer desta forma, concentradas em 3 momentos, e sim baseadas nos índices de disponibilidade. De acordo com o histórico de quebras da embaladora à vácuo no período PRÉ-MCC, no ano de 2019 a embaladora à vácuo falhou 27 vezes, ocasionando um custo total de manutenção de $\mathrm{R} \$ 107.855,00$, ficando mais de $650 \mathrm{~h}$ sem produzir, em virtude de paradas não programadas, com perdas por lucro cessante estimado em mais de $\mathrm{R} \$ 4.000 .000,00$.

\subsection{Resultados período PÓS-MCC}

Após a análise dos indicadores e dados do período PRÉ-MCC, onde as atividades se resumiam em manutenção preventiva mecânica, foi elaborado o novo plano de manutenção com base na metodologia MCC. A Fig. 5 apresenta um excerto do plano de manutenção PÓS-MCC. Foram criadas e otimizadas atividades de manutenção preventiva mecânica, elétrica, preditiva mecânica, elétrica, de lubrificação e de inspeção de rota (destaque da Fig. 5), já com os tempos estimados e indicados no resultado. Esse plano de manutenção foi criado de maneira a facilitar a atualizações futuras e organizar programação para cada tipo de manutentor na atividade, onde temse diferentes equipes executando cada tipo de manutenção. Com base na metodologia MCC, reduziram-se as atividades tratadas no plano de manutenção PRÉ-MCC, otimizando e separando as atividades por categorias.

\begin{tabular}{|c|c|c|c|c|c|c|c|c|c|c|c|}
\hline $\begin{array}{l}\text { Equipan } \\
\text { GrpLisT }\end{array}$ & SELADOP & & & & & & & & & & \\
\hline \multicolumn{12}{|c|}{ Sínteqe geral roteiros } \\
\hline NGr & TxtBreve roteiro & Cen. & MarEl & Estratég. & Utilização & GrPI. & Status & C & Conjunto & PCtrl & NE \\
\hline 1 & 10008177 - PR - MECANICA & FABR & $\square$ & VRBMAQ & 4 & 1 & 2 & 1 & & & Numeraçi • \\
\hline 2 & 10008177 - PR - ELETRICA & FABR & $\square$ & VRBMAQ & 4 & 1 & 2 & 1 & & & Numeraçi ${ }^{-}$ \\
\hline 3 & 10008177 - PD - ELETRICA & FABR & $\square$ & VRBMAQ & 4 & 1 & 2 & 1 & & & Numeraçi \\
\hline 4 & 10008177 - PD - MECANICA & FABR & $\square$ & VRBMAQ & 4 & 1 & 2 & 1 & & & Numeraçi \\
\hline 5 & 10008177-LU-LUBRIFICACAO & FABR & $\square$ & VRBMAQ & 4 & 1 & 4 & 1 & & & Numeraçi $L$ \\
\hline 6 & 10008177-IR-INSP. ROTA & FABR & $\square$ & VRBMAQ & 4 & 1 & 4 & 1 & & & Numeraçi \\
\hline
\end{tabular}

Figura 5 - Excerto do plano de manutenção PÓS-MCC.

A Fig. 6 apresenta o excerto do calendário de troca do plano de manutenção PÓS-MCC. Observa-se que o tempo de troca preventiva, de acordo com o resultado do estudo, foi atualizado. Com as trocas executadas de acordo com estes períodos garante-se a confiabilidade inicialmente estipulada. Foram criadas novas listas de tarefas de manutenções preventiva elétrica, preditiva mecânica, preditiva elétrica, inspeção de rota e de lubrificação.

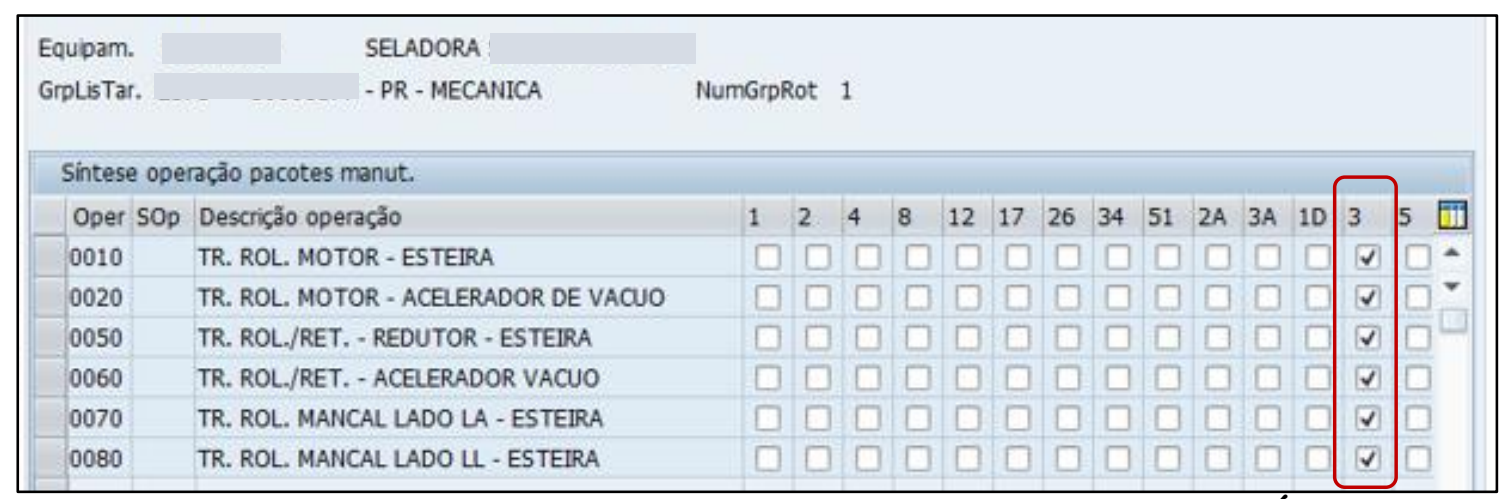

Figura 6 - Excerto do calendário de trocas: plano de manutenção PÓS-MCC.

Após a criação e aplicação do novo plano de manutenção com base na metodologia MCC foram mensurados os índices de disponibilidade e confiabilidade da embaladora a vácuo no período de janeiro a março de 2020. A Fig. 7 apresenta os resultados dos índices de disponibilidade nos períodos PRÉ-MCC e PÓS-MCC. Observa-se nesta figura uma estabilização do índice de disponibilidade após a aplicação do novo plano de manutenção. Deve-se salientar que para manter 
confiabilidade se deve intervir na máquina em algumas tarefas, o que interrompe a produção. Por conta destas paradas de máquina o índice de disponibilidade oscilou entre $94 \%$ e $97 \%$.

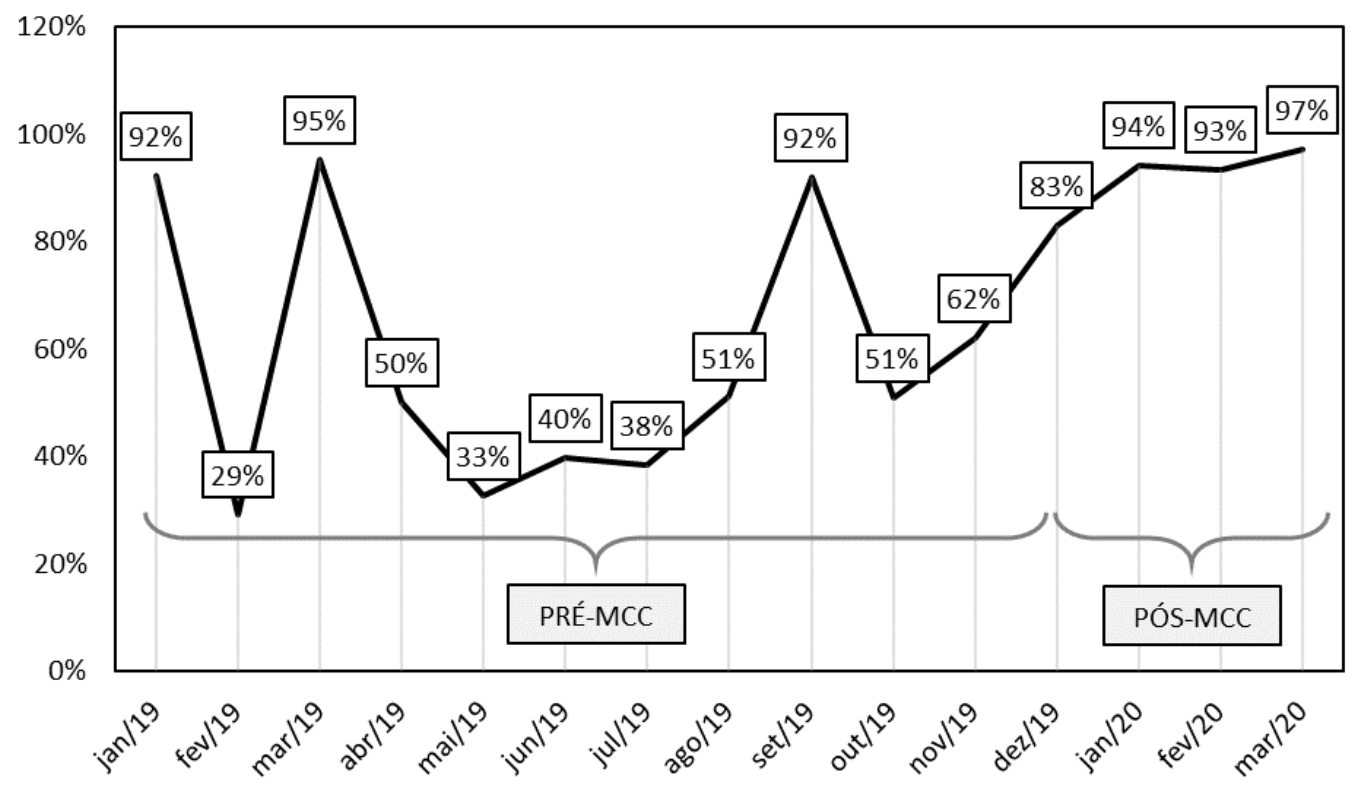

Figura 7 - Índice de disponibilidade nos períodos PRÉ-MCC e PÓS-MCC.

A Fig. 8 mostra a evolução do índice de confiabilidade nos períodos PRÉ-MCC e PÓSMCC. De acordo com o índice de confiabilidade inicialmente estipulado, a máquina não pode falhar em 3.000 h para manter o nível de confiabilidade acima de $80 \%$. Durante o período PÓS-MCC (Fig. 8) os itens tratados da embaladora à vácuo não manifestaram falha, comprovando que a metodologia MCC traz resultados significativos na gestão dos ativos e da manutenção industrial alimentícia. Isto é verificado no aumento do índice de confiabilidade de $58 \%$ para $81 \%$ no período PÓS-MCC.

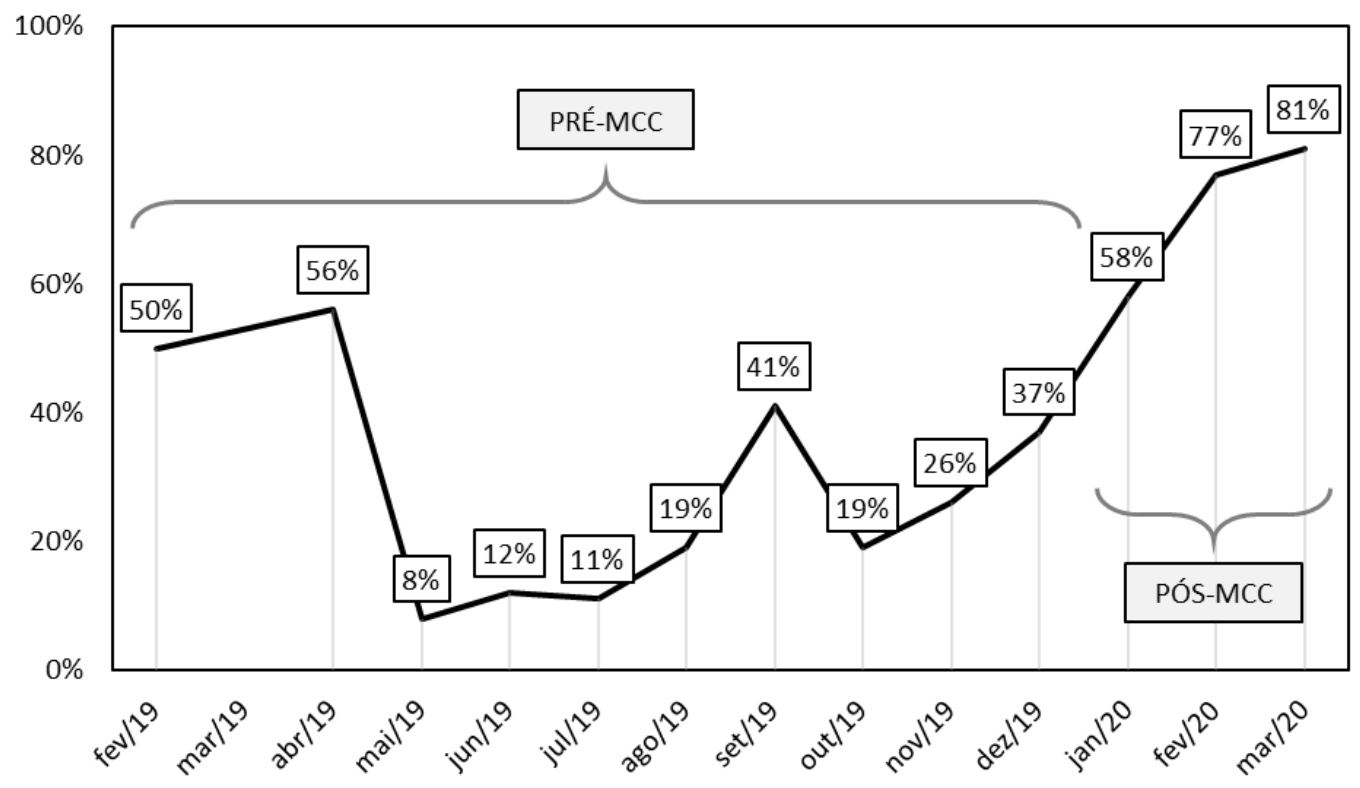

Figura 8 - Índice de confiabilidade nos períodos PRÉ-MCC e PÓS-MCC.

A Tab. 10 apresenta os custos de manutenção dos períodos PRÉ-MCC e PÓS-MCC. Nesta tabela é possível verificar que houve uma redução nos custos efetivo de manutenção de $57 \%$ nos meses apurados com o emprego da metodologia MCC na embaladora à vácuo. Como os índices do período PÓS-MCC compreenderam somente 3 meses, a extrapolação aponta que a redução dos 
custos efetivos de manutenção poderá chegar a 40\%. Comparando-se os primeiros quadrimestres de 2019 e 2020 na Tab. 10, se observa uma redução dos custos de perdas de produção em torno de $74 \%$, um aumento da disponibilidade da máquina para índices acima de $92 \%$, com redução significativa de paradas de máquina para manutenções não programadas.

Tabela 10 - Tabela de comparação de custos de manutenção 2019/2020.

\begin{tabular}{c|c|c|c|c|c}
\hline Período & Disponib. & $\begin{array}{c}\text { Custo com } \\
\text { manutenção [R\$] }\end{array}$ & Período & Disponib. & $\begin{array}{c}\text { Custo com } \\
\text { manutenção [R\$] }\end{array}$ \\
\hline jan/19 & $92 \%$ & $16.500,00$ & jan/20 & $94 \%$ & $5.370,00$ \\
\hline fev/19 & $29 \%$ & $8.250,00$ & $\mathrm{fev} / 20$ & $93 \%$ & $5.370,00$ \\
\hline mar/19 & $95 \%$ & $5.300,00$ & $\mathrm{mar} / 20$ & $97 \%$ & $5.370,00$ \\
\hline abr/19 & $50 \%$ & $20.050,00$ & $\mathrm{abr} / 20$ & $0 \%$ & $5.370,00$ \\
\hline mai/19 & $33 \%$ & $12.750,00$ & $\mathrm{mai} / 20$ & $0 \%$ & $5.370,00$ \\
\hline jun/19 & $40 \%$ & $13.100,00$ & $\mathrm{jun} / 20$ & $0 \%$ & $5.370,00$ \\
\hline jul/19 & $38 \%$ & $10.000,00$ & jul/20 & $0 \%$ & $5.370,00$ \\
\hline ago/19 & $51 \%$ & $8.900,00$ & $\mathrm{ago} / 20$ & $0 \%$ & $5.370,00$ \\
\hline set/19 & $92 \%$ & $3.900,00$ & $\mathrm{set} / 20$ & $0 \%$ & $5.370,00$ \\
\hline out/19 & $51 \%$ & $3.305,00$ & out/20 & $0 \%$ & $5.370,00$ \\
\hline nov/19 & $62 \%$ & $5.800,00$ & nov/20 & $0 \%$ & $5.370,00$ \\
\hline dez/19 & $83 \%$ & $5.370,00$ & dez/20 & $0 \%$ & $5.370,00$ \\
\hline \multicolumn{7}{c|}{ Total PRÉ-MCC } & $\mathbf{1 0 7 . 8 5 5 , 0 0}$ & Total PÓS-MCC & $\mathbf{6 4 . 4 4 0 , 0 0}$ \\
\hline
\end{tabular}

A Tab. 11 apresenta os custos efetivos de manutenção mensais dos períodos PRÉ-MCC e PÓS-MCC. Fica evidente a redução significativa dos custos de manutenção e aumento do lucro da empresa, demonstrando que metodologia MCC contribuiu fortemente com lucratividade da empresa de maneira direta ao reduzir os gastos com manutenção. Os resultados da Tab. 10 e Tab. 11 corroboram com as afirmações de Kardec e Nascif (2009), os quais indicam que a MCC possibilita a diminuição dos custos de manutenção, aumento dos lucros através da redução de paradas não programadas, aumento da confiabilidade dos equipamentos, um maior conhecimento sobre as maquinas e uma maior interação entre profissionais da manutenção na busca por soluções de problemas de máquinas.

Tabela 11 - Custos de manutenção mensais: períodos PRÉ-MCC e PÓS-MCC.

\begin{tabular}{c|c|c|c}
\hline \multicolumn{4}{c}{ REDUÇÃO DE CUSTO/MÊS } \\
\hline & PRÉ-MCC & PÓS-MCC & Melhoria \\
\hline Tempo de parada por manutenção [h] & 55,5 & 14 & 41,5 \\
\hline Quantidade não produzida [kg] & $131.630,00$ & $33.264,00$ & $98.366,00$ \\
\hline Lucro cessante no período [R\$] & $250.097,00$ & $63.201,60$ & $186.895,40$ \\
\hline
\end{tabular}

Na Tab. 11 constata-se que no período PRÉ-MCC com o tempo de 55,4 h de máquina parada a empresa deixou de produzir mais de 130 t, ocasionando um prejuízo em torno de $\mathrm{R} \$ 250.000,00$. Com a aplicação da metodologia reduziu-se este tempo em $41 \mathrm{~h}$ de máquina parada, diminuindo o prejuízo em R \$186.800,00. 


\section{Conclusões}

Este trabalho abordou a aplicação da metodologia MCC em uma embaladora à vácuo de uma linha de produção do setor alimentício. Foram coletadas as informações do equipamento, analisado os modos e efeitos de falha, seleção de funções significantes e avaliadas as atividades aplicáveis. Com os resultados atingidos neste trabalho conclui-se que:

- Aplicação da metodologia MCC contribuiu para o aumento da confiabilidade, que não superava $56 \%$, passando dos $80 \%$. Isto permite que embaladora à vácuo seja melhor explorada no âmbito de produção, que o planejamento de produção seja mais seguro e que os compromissos com entregas dos produtos embalados sejam cumpridos;

- A aplicação da metodologia MCC contribuiu significativamente para o aumento da disponibilidade da embaladora a vácuo. Esse índice passou de uma média de 55\% para acima dos $92 \%$ após a aplicação da MCC. Isso apresenta que grande parte do tempo disponível da máquina não era utilizado por conta de paradas com manutenção não planejadas;

- A aplicação da metodologia MCC permitiu uma economia de recursos de mais $\mathrm{R} \$ 280.000,00$. Para a empresa isso implica em uma linha de produção mais eficiente e lucrativa;

- A coleta dos dados referentes aos indicadores de confiabilidade e disponibilidade deve ser bem planejada e padronizada pelos gestores da manutenção antes da aplicação da metodologia MCC. Os dados coletados, referentes aos indicadores, devem ser revisitados constantemente, afim de reduzir ainda mais os custos de manutenção, uma vez que problemas podem surgir na linha de produção

\section{Referências}

Alkaim, J. L. (2003) Metodologia para incorporar conhecimento intensivo às tarefas de Manutenção Centrada na Confiabilidade aplicada em ativos de sistemas elétricos. Tese de doutorado, Universidade Federal de Santa Catarina, Florianópolis, SC, Brasil.

Amaral, B. F. (2017) Manutenção centrada em confiabilidade: estudo de caso da implementação da ferramenta MCC pela Transpetro. Trabalho de Conclusão de Curso, Centro de Instrução Almirante Graça Aranha, Marinha do Brasil, Rio de Janeiro, RJ, Brasil.

Araldi, V. E.; Bonamigo, A; Vanin A. B. \& Azzolini, J. C. (2019). Proposta de um plano de manutenção centrada na confiabilidade para uma máquina de usinagem controlada por comando numérico computadorizado (CNC), IX Congresso Brasileiro de Engenharia de Produção, Ponta Grossa, Brasil.

Bandeira, H. C.; Costa, D. O. \& Lopes, R. S. (2016). Estudo da manutenção centrada em confiabilidade aplicada a um sistema de refrigeração. XXXVI Encontro Nacional de Engenharia de Produção, João Pessoa, Brasil.

Baran, L. R. (2011) Manutenção centrada em confiabilidade aplicada na redução de falhas: um estudo de caso. Monografia de especialização, Universidade Federal Tecnológica Federal do Paraná, Ponta Grossa, PR, Brasil.

Belinelli, M. M. (2015) Desenvolvimento de método para seleção de política de lubrificação de máquinas centrada em confiabilidade: aplicação na indústria alimentícia. Tese de doutorado, Escola Politécnica da USP, São Paulo, SP, Brasil.

Caiado, R. G. G.; Lima, G. B. A. \& Quelhas, O. L. G. (2015). Aspectos da aplicação da manutenção centrada em confiabilidade. IX Congresso Nacional de Excelência em Gestão, Niterói, Brasil.

Fonseca, R. R., Franco, I. C., \& Silva, F. V. da. (2016). Bioreactor temperature control using a generic fuzzy feedforward control system. 841: Intelligent Systems and Control. Modelling, Simulation and Identification / 841: Intelligent Systems and Control, Campinas, Brazil. https://doi.org/10.2316/P.2016.841-024 
Cerveira, D. S. \& Sellitto, M. A. (2015). Manutenção centrada em confiabilidade (MCC): análise quantitativa de um forno elétrico a indução. Revista Produção Online, (2), 405-432. doi: https://doi.org/10.14488/1676-1901.v15i2.1615.

CORREIA JUNIOR, W. Matriz GUT: saiba como priorizar seus problemas, 2016. Disponível em: <https://empreendadentista.com.br/2016/04/15/matriz-gut/>. Acesso em janeiro de 2020.

Drumond, M. R. (2004). Manual do sistema de gerenciamento da manutenção: Companhia Vale do Rio Doce. Minas Gerais.

Fleming, P. V.; Silva, F. F. \& França, S. R. R. O. (1999). Aplicando manutenção centrada em confiabilidade (MCC) em indústrias brasileiras: lições aprendidas. Encontro Nacional de Engenharia de Produção, Rio de Janeiro, Brasil.

Fogliatto, F. S. \& Ribeiro, J. L. D. Confiabilidade e Manutenção Industrial. Campus - Elsevier.

IEC 60300-3-11:2009. Dependability management - Part 3-11: Application guide - Reliability centred maintenance International Electrotechnical Commission's. Switzerland.

Kardec, A. \& Nascif, J. Manutenção: Função Estratégica. 3. ed. Rio de Janeiro: Qualitymark, 2009.

Lucatelli, M. V. (2012). Proposta de aplicação da manutenção centrada em confiabilidade em equipamentos médico-hospitalares. Tese de doutorado, Universidade Federal de Santa Catarina, Florianópolis, SC, Brasil

Mendes, A. A. (2011). Manutenção Centrada em Confiabilidade: uma abordagem quantitativa. Dissertação de mestrado, Universidade Federal do Rio Grande do Sul, Porto Alegre, RS, Brasil.

Moubray, J. (1997). Reliability-centered maintenance: second edition. $2^{a}$. ed. New York: Industrial Press Inc.

Pereira, T. A. (2015). Manutenção Centrada na Confiabilidade: aplicação da metodologia da Manutenção Centrada na Confiabilidade em uma embaladora automática vertical. Trabalho de Conclusão de Curso, UNIS, Varginha, MG, Brasil.

Rosa, R. N. (2016) Aplicação da manutenção centrada em confiabilidade em um processo da indústria automobilística. Dissertação de mestrado. Universidade Federal do Rio Grande do Sul, Porto Alegre, RS, Brasil.

SAE JA1011. (1999). Evaluation Criteria for Reliability Centered Maintenance (RCM) Processes. Society of Automotive Engineers.

Sellitto, M. A.; Borchardt, M. \& Araújo D. R. C. (2002). Manutenção centrada em confiabilidade: aplicando uma abordagem quantitativa. XXII Encontro Nacional de Engenharia de Produção, Curitiba, Brasil.

Silva, E. B. (2018). Manutenção centrada em confiabilidade visando a competividade em uma indústria moedora de grãos na região dos campos gerais. Trabalho de Conclusão de Curso. Universidade Federal Tecnológica do Paraná, Ponta Grossa, PR, Brasil.

Silva, R. P. (2004). Gerenciamento do setor de manutenção. Monografia de especialização. Universidade de Taubaté, Taubaté, SP, Brasil.

Siqueira, Y. P. D. S. (2014). Manutenção Centrada em Confiabilidade: Manual de Implementação 3. ed. Quallitymark.

Siqueira, Y. P. D. S. (2014). Manutenção centrada na confiabilidade: Manual de implantação. $1^{a}$ ed. Qualitymark.

Smith, A. M. \& Hinchcliffe, G. R. (2004). RCM: Gateway to world class maintenance. $2^{\mathrm{a}}$. ed. Burlington: Elsevier Butterworth-Heinemann, v.

TELES, J. FMEA: O que e como fazer. Engeteles, 2017. Disponível em: https://engeteles.com.br/fmea-o-que-e-como-fazer/ 2019 Acesso em novembro de 2019.

Wilmeth, G. W. \& Usrey, M. W. (2000). Reliability centered maintenance: a case study, Engineering Management Journal, 12(4), 25-31, $2000 . \quad$ doi: https://doi.org/10.1080/10429247.2000.11415089. 